\title{
CONVERGENCE OF WEIGHTED SUMS OF INDEPENDENT RANDOM VARIABLES AND AN EXTENSION TO BANACH SPACE-VALUED RANDOM VARIABLES
}

\author{
W.J. PADGETT and R.L. TAYLOR \\ Department of Mathematics, Computer Science, and Statistics \\ University of South Carolina, Columbia \\ Columbia, South Carolina 29208 U.S.A.
}

(Received June 23, 1978)

ABSTRACT. Let $\left\{\mathrm{X}_{k}\right\}$ be independent random variables with $\mathrm{EX}_{k}=0$ for all $k$ and let $\left\{a_{n k}: n \geq 1, k \geq 1\right\}$ be an array of real numbers. In this paper the almost sure convergence of $s_{n}=\sum_{k=1}^{n} a_{n k} x_{k}, n=1,2, \ldots$, to a constant is studied under various conditions on the weights $\left\{a_{n k}\right\}$ and on the random variables $\left\{\mathrm{X}_{\mathrm{k}}\right\}$ using martingale theory. In addition, the results are extended to weighted sums of random elements in Banach spaces which have Schauder bases. This extension provides a convergence theorem that applies to stochastic processes which may be considered as random elements in function spaces.

KEY WORDS AND PHRASES. Weighted Sums, Strong Law of Large Numbers, Almost Sure Convergence, Generalized Gaussian Random Variables, Random Elements in Banach Space, Schauder Basis.

AMS (MOS) SUBJECT CLASSIFICATION (1970) CODES. Primary 60F15, 60B05; Secondary 60699. 


\section{INTRODUCTION.}

Let $(\Omega, F, P)$ denote a probability space and let $\left\{x_{k}\right\}$ be a sequence of independent random variables with $\mathrm{EX}_{\mathrm{k}}=0$ for all $\mathrm{k}=1,2, \ldots$. Let $\left\{a_{n k}: n \geq 1, k \geq 1\right\}$ be an array of real numbers and define $s_{n}=\sum_{k=1}^{n} a_{n k} x_{k}$, $\mathrm{n}=1,2, \ldots$.

Several results have been obtained in recent years concerning the almost sure convergence of the sequence $\left\{s_{n}\right\}$ under various conditions on the weights $\left\{a_{n k}\right\}$ and boundedness conditions on the random variables $\left\{x_{k}\right\}$ or on their moments. For example, Chow (1966) and Stout (1968) required conditions on $\left\{a_{n k}\right\}$ such as (1) $\sum_{k=1}^{\infty} a_{n k}^{2} \leq K n^{\gamma}$ for some constants $K$ and $\gamma$ or (ii) $A_{n}=\sum_{k=1}^{\infty} a_{n k}^{2}<\infty$ for all $n$ and $\sum_{n=1}^{\infty} \exp \left(-\frac{\lambda}{A_{n}}\right)<\infty$ for all $\lambda>0$, and either a uniform bound on certain moments of the random variables $\left\{x_{k}\right\}$ or the condition that for all $k, E\left[\exp \left(t x_{k}\right)\right] \leq \exp \left(\alpha^{2} \tau^{2} / 2\right)$ for some $\alpha \geq 0$ and all real numbers t. Stout's (1968) results concerned the complete convergence of $\left\{\mathrm{S}_{\mathrm{n}}\right\}$ in the sense of Hsu and Robbins (1947) which implies almost sure convergence. Also, Rohatgi (1971) considered the almost sure convergence of $\left\{s_{n}\right\}$ to zero by requiring the uniform dominance of $\left\{x_{k}\right\}$ in the sense that there exists a random variable $X$ such that $P\left[\left|x_{k}\right| \geq a\right] \leq P[|x| \leq a]$ for all $a>0$ and all $\mathrm{k}$, where $\mathrm{X}$ has a finite $(1+1 / \alpha)$ th absolute moment and $\alpha>0$ is such that $\max \left|a_{n k}\right|=0\left(n^{-\alpha}\right)$. (It was also assumed that $\lim _{n \rightarrow \infty} a_{n k}=0$ for all $k$ and $\sum_{k=1}^{\infty}\left|a_{n k}\right| \leq C$ for all n.) More recently, Chow and Lai (1973) have studied

the almost sure convergence of $\left\{n^{-\frac{1}{\alpha}} S_{n}\right\}$ (some $1 \leq \alpha \leq 2$ ) for independent and identically distributed random variables with finite ath absolute moments and with somewhat weaker conditions on the array $\left\{a_{n k}\right\}$ than those in Stout (1968). Lai (1974) has indicated the importance of weighted sums in control charts. 
Chow (1966) defined a random variable $\mathrm{X}$ to be generalized Gaussian if there exists a number $\alpha \geq 0$ (referred to here as the parameter) such that for every real number $t, E[\exp (t x)] \leq \exp \left(\alpha^{2} t^{2} / 2\right)$. Special cases of generalized Gaussian random variables are normal with zero means or bounded, symmetric random variables.

In Section 2 of this paper the definition of a totally generalized Gaussian random variable is given. Then the almost sure convergence of $\left\{\mathrm{s}_{n}\right\}$ for independent (but not necessarily identically distributed) random variables which are totally generalized Gaussian is obtained using martingale theory with very general conditions on the sequence of weights $\left\{a_{n k}\right\}$. The relaxation of the restrictions on the weights which is achieved in these results is summarized and discussed in Section 3. Also, in Section 4 the results for random variables are extended to sequences of independent random elements in separable Banach spaces. This extension will provide a convergence theory for weighted sums of stochastic processes which may be considered as random elements in function spaces, such as the Wiener process on a closed interval $[0, \mathrm{~T}]$ for some $\mathrm{T}>0$ [see Billingsley (1968)].

2. WEIGHTED SUMS OF RANDOM VARIABLES.

Let $\left\{a_{n k}: n \geq 1, k \geq 1\right\}$ be a double sequence of real numbers satisfying the following conditions:

(1) $a_{n k} \geq 0$ for all $k$ and $n, a_{n-1, k} \geq a_{n k}$ for $k=1,2, \ldots, n-1$ and $\sum_{k=n}^{\infty} a_{n-1, k}^{2} \geq \sum_{k=n}^{\infty} a_{n k}^{2}$ for all $n$;

(2) $\sum_{k=1}^{\infty} a_{n k}^{2} \leq \Gamma$ for all $n$ and some positive constant $\Gamma$;

(3) $\sum_{k=n+1}^{\infty} a_{n k}^{2} \rightarrow 0$ as $n \rightarrow \infty$; and

(4) $\lim _{n \rightarrow \infty} a_{n k}=0$ for all k. 
A sequence $\left\{a_{n k}\right\}$ satisfying Conditions (1)-(4) will be called a Type (P) sequence.

Conditions (1)-(4) will be considered in detail in Section 3, and several examples of sequences which are of Type (P) will be given there. Those examples will contrast previous results with the results which are given in this section and will show that the weights can be very different from the traditional weights $a_{n k}=n^{-1}$ if $k=1, \ldots, n$. However, in Section 3 , the traditional weighting is shown to be a special case of a Type (P) sequence by letting $a_{n k}=k^{-3 / 4}$ if $k=n+1, \ldots$.

DEFINITION 1. Let $\mathrm{X}$ be a random variable such that its expected value EX exists. Let $I_{A}$ be the indicator random variable for the event $A$, and let $\mathrm{X}^{+}=\mathrm{XI}_{[\mathrm{X} \geq 0]}$ and $\mathrm{X}^{-}=-\mathrm{XI}_{[\mathrm{X}<0]^{-}}$Then $\mathrm{X}$ will be said to be totally generalized Gaussian if $\mathrm{X}^{+}-\mathrm{EX}^{+}$is generalized Gaussian with parameter $\alpha \leq 2^{\frac{1}{2}}$ and $\mathrm{X}^{-}-\mathrm{EX}^{-}$is generalized Gaussian with parameter $\beta \leq 2^{\frac{1}{2}}$.

We now prove the following theorem.

THEOREM 1. Let $\left\{\mathrm{X}_{\mathrm{k}}\right\}$ be independent random variables with $\mathrm{EX}_{\mathrm{k}}=0$ for each $k$ and let $\left\{a_{n k}\right\}$ be a Type (P) sequence. If for each $k$, $x_{k}$ is totally generalized Gaussian, then there exists a constant $\gamma$ such that as $n \rightarrow \infty$

$$
s_{n}=\sum_{k=1}^{n} a_{n k} x_{k} \rightarrow r \text { almost surely. }
$$

PROOF. For each $k$, let $\mathrm{x}_{\mathrm{k}}^{+}$and $\mathrm{x}_{\mathrm{k}}^{-}$be defined as in Definition 1 . Then $\mathrm{x}_{\mathrm{k}}=\mathrm{x}_{\mathrm{k}}^{+}-\mathrm{x}_{\mathrm{k}}^{-}, \mathrm{k}=1,2, \ldots$. Also, define $F_{\mathrm{n}}^{+}=\sigma\left\{\mathrm{x}_{1}^{+}, \ldots, \mathrm{x}_{\mathrm{n}}^{+}\right\}$to be

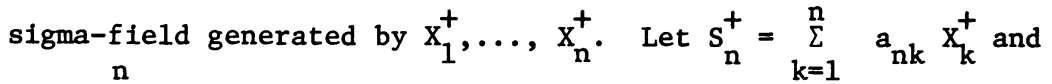
$s_{n}^{-}=\sum_{k=1}^{n} a_{n k} x_{k}^{-}$for $n=1,2, \ldots$. Now, $\left\{\mathrm{x}_{\mathrm{k}}^{+}\right\}$are independent random variables, and by hypothesis we may obtain for every $k$

$$
\begin{aligned}
E\left[\exp \left(t X_{k}^{+}\right)\right] & =E\left\{\exp \left[t\left(X_{k}^{+}-E_{k}^{+}\right)\right] \exp \left(t E_{k}^{+}\right)\right\} \\
& \leq \exp \left(t^{2}+t E X_{k}^{+}\right)
\end{aligned}
$$


for all real numbers $t$. Define

$$
\mathrm{Y}_{\mathrm{n}}^{+}=\exp \left(\mathrm{S}_{\mathrm{n}}^{+}+\sum_{\mathrm{k}=\mathrm{n}+1}^{\infty} \mathrm{a}_{\mathrm{nk}}^{2}-\sum_{\mathrm{k}=1}^{\mathrm{n}} \mathrm{a}_{\mathrm{kk}} \mathrm{EX}_{\mathrm{k}}^{+}\right), \quad \mathrm{n}=1,2, \ldots
$$

Then for $n \geq 2$ by Condition (1) on $\left\{a_{n k}\right\}$ and inequality (2.1) we obtain

$$
\begin{aligned}
& E\left[Y_{n}^{+} \mid F_{n-1}^{+}\right]=E\left[\exp \left(S_{n}^{+}+\sum_{k=n+1}^{\infty} a_{n k}^{2}\right) \mid F_{n-1}^{+}\right] \exp \left(-\sum_{k=1}^{n} a_{k k} \quad E x_{k}^{+}\right) \\
& =E\left[\exp \left(\sum_{k=1}^{n-1} a_{n k} x_{k}^{+}+a_{n n} x_{n}^{+}+\sum_{k=n+1}^{\infty} a_{n k}^{2}\right) \mid F_{n-1}^{+}\right] \\
& \times \exp \left(-\sum_{k=1}^{n} a_{k k} E X_{k}^{+}\right) \\
& =\exp \left(\sum_{k=1}^{n-1} a_{n k} x_{k}^{+}+\sum_{k=n+1}^{\infty} a_{n k}^{2}\right) E\left[\exp \left(a_{n n} x_{n}^{+}\right)\right] \exp \left(-\sum_{k=1}^{n} a_{k k} E x_{k}^{+}\right) \\
& \leq \exp \left(\sum_{k=1}^{n-1} a_{n k} x_{k}^{+}+\sum_{k=n+1}^{\infty} a_{n k}^{2}\right) \exp \left(a_{n n}^{2}+a_{n n} \operatorname{Ex}_{n}^{+}\right) \\
& \times \exp \left(-\sum_{k=1}^{n} a_{k k} E x_{k}^{+}\right) \\
& =\exp \left(\sum_{k=1}^{n-1} a_{n k} x_{k}^{+}+\sum_{k=n}^{\infty} a_{n k}^{2}\right) \exp \left(-\sum_{k=1}^{n-1} a_{k k} E x_{k}^{+}\right) \\
& \leq \exp \left(\sum_{k=1}^{n-1} a_{n-1, k} x_{k}^{+}+\sum_{k=n}^{\infty} a_{n-1, k}^{2} \sum_{k=1}^{n-1} a_{k k} \operatorname{Ex}_{k}^{+}\right) \\
& =\mathrm{Y}_{\mathrm{n}-1}^{+} \quad \text { a.s. }
\end{aligned}
$$

Hence, $\left\{\mathrm{Y}_{\mathrm{n}}^{+}\right\}$is a supermartingale with respect to $\left\{F_{n}^{+}\right\}$.

For each $\mathbf{n}$

$$
\begin{aligned}
E\left|Y_{n}^{+}\right| & =E\left[\exp \left(s_{n}^{+}+\sum_{k=1}^{\infty} a_{n k}^{2}-\sum_{k=1}^{n} a_{k k} E x_{k}^{+}\right)\right] \\
& \left.=\underset{k=1}{[\Pi} E\left[\exp \left(a_{n k} x_{k}^{+}\right)\right]\right] \exp \left(\sum_{k=n+1}^{\infty} a_{n k}^{2}-\sum_{k=1}^{n} a_{k k} E x_{k}^{+}\right) \\
& \left.\leq \underset{k=1}{n} \exp \left(a_{n k}^{2}+a_{n k} E x_{k}^{+}\right)\right] \exp \left(\sum_{k=n+1}^{\infty} a_{n k}^{2}-\sum_{k=1}^{n} a_{k k} E x_{k}^{+}\right) \\
& =\exp \left(\sum_{k=1}^{\infty} a_{n k}^{2}\right) \exp \left[\sum_{k=1}^{n}\left(a_{n k}-a_{k k}\right) E x_{k}^{+}\right] \\
& \leq \exp (\Gamma)<\infty
\end{aligned}
$$


by inequality (2.1) and Conditions (1) (that $a_{n-1, k} \geq a_{n k}$ for $k=1,2, \ldots, n-1$ and (2) on the $\left\{a_{n k}\right\}$. Thus, sup $E\left|Y_{n}^{+}\right|<\infty$, and by the martingale convergence theorem [Tucker (1967)], there is a random variable $Z_{1}^{\prime}$ such that $\mathrm{Y}_{\mathrm{n}}^{+} \rightarrow \mathrm{Z}_{1}^{\prime}$ a.s. By Condition (3) of a Type (P) sequence, there exists a random variable $z_{1}$ such that $\mathrm{s}_{\mathrm{n}}^{+}-\Sigma_{\mathrm{k}=1}^{\mathrm{n}} \quad \mathrm{a}_{\mathrm{kk}} \operatorname{Ex}_{\mathrm{k}}^{+} \rightarrow z_{1}$ a.s. Similar1y, there exists a random variable $z_{2}$ such that $S_{n}^{-}-\sum_{k=1}^{n} \quad a_{k k}{E X_{k}^{-} \rightarrow Z_{2}}^{a}$.s. Next, $\operatorname{EX}_{k}=0$ implies that $\mathrm{EX}_{\mathrm{k}}^{+}=\mathrm{EX}_{\mathrm{k}}^{-}$. Hence, $\mathrm{s}_{\mathrm{n}}=\mathrm{s}_{\mathrm{n}}^{+}-\mathrm{s}_{\mathrm{n}}^{-}=$ $\left(S_{n}^{+}-\Sigma_{k=1}^{n} \quad a_{k k} E X_{k}^{+}\right)-\left(S_{n}^{-}-\Sigma_{k=1}^{n} \quad a_{k k} E X_{k}^{-}\right) \rightarrow z_{1}-z_{2}=z$ a.s.

By Condition (4) of Type (P) sequence, for each $j=1,2, \ldots, a_{n j} x_{j} 0$ a.s. as $n \rightarrow \infty$. Therefore, $s_{n}=\left(a_{n 1} x_{1}+\ldots+a_{n, k-1} x_{k-1}\right)+\left(a_{n k} x_{k}+\ldots\right.$ $\left.+a_{n n} x_{n}\right) \rightarrow z$ a.s. implies that for every $k, \sum_{j=k}^{n} a_{n j} x_{j} \rightarrow z$ a.s. Thus, $Z$ is measurable with respect to the tail sigma-field of $\left\{x_{k}\right\}$ and there exists a constant $\gamma$ such that $P[z=\gamma]=1$, Tucker (1967, p. 75). Therefore, $s_{n} \rightarrow \gamma$ a.s. ///

If, in addition to the Conditions (1)-(4), the sequence $\left\{a_{n k}\right\}$ satisfies the condition that $\sum_{k=1}^{n} a_{n k}^{2} \rightarrow 0$ as $n \rightarrow \infty$, then the following theorem may be obtained.

THEOREM 2. Let $\left\{x_{k}\right\}$ be a sequence of independent random variables with $\mathrm{EX}_{\mathrm{k}}=0$ for all $\mathrm{k}$, and let $\left\{\mathrm{a}_{\mathrm{nk}}\right\}$ be a Type (P) sequence. If $\mathrm{X}_{k}$ is totally generalized Gaussian for each $k$, and if $\sum_{k=1}^{n} a_{n k}^{2} \rightarrow 0$ as $n \rightarrow \infty$, then $s_{n}=\sum_{k=1}^{n} a_{n k} x_{k} \rightarrow 0$ almost surely as $n \rightarrow \infty$.

PROOF. By Theorem 1, there exists a constant $\gamma$ such that $s_{n} \rightarrow \gamma$ almost surely. Hence, $s_{n} \rightarrow \gamma$ in probability. Let $s_{n}^{+}$and $s_{n}^{-}$be defined as in the proof of Theorem 1. Define $\mu_{n}^{+}=\operatorname{ES}_{n}^{+}=\sum_{k=1}^{n} a_{n k} \operatorname{EX}_{k}^{+}$. By Chebyshev's inequality for $\epsilon>0$ 


$$
\left.P\left[\mid S_{n}^{+}-\mu_{n}^{+}\right\}>\epsilon\right] \leq \epsilon^{-2} \operatorname{var}\left(S_{n}^{+}\right)=\epsilon^{-2} \sum_{k=1}^{n} a_{n k}^{2} \operatorname{var}\left(x_{k}^{+}\right) .
$$

Since $\mathrm{X}_{\mathrm{k} 0}^{+}=\mathrm{X}_{\mathrm{k}}^{+}-\mathrm{EX}_{\mathrm{k}}^{+}$is generalized Gaussian with $\alpha_{\mathrm{k}}^{2} \leq 2$ and mean zero, $\operatorname{var}\left(\mathrm{X}_{\mathrm{k}}^{+}\right)=\mathrm{E}\left(\mathrm{X}_{\mathrm{k} 0}^{+}\right)^{2}$ is uniformly bounded for all $\mathrm{k}$ by a positive constant $B$. Thus,

$$
\mathrm{P}\left[\left|\mathrm{s}_{\mathrm{n}}^{+}-\mu_{\mathrm{n}}^{+}\right|>\epsilon\right] \leq \epsilon^{-2} \cdot \mathrm{B} \sum_{\mathrm{k}=1}^{\mathrm{n}} \mathrm{a}_{\mathrm{nk}}^{2} \rightarrow 0 \text { as } \mathrm{n} \rightarrow \infty \text { by hypothesis. }
$$

That is, $s_{n}^{+}-\mu_{n}^{+} \rightarrow 0$ in probability as $n \rightarrow \infty$.

The same argument gives $s_{n}^{-}-\mu_{n}^{-} \rightarrow 0$ in probability as $n \rightarrow \infty$. Hence, combining the two results yields $s_{n}=\left(s_{n}^{+}-\mu_{n}^{+}\right)-\left(s_{n}^{-}-\mu_{n}^{-}\right) \rightarrow 0$ in probability as $\mathrm{n} \rightarrow \infty$. This implies that $\gamma=0$ since the limit in probability is unique. That is, $\mathrm{s}_{\mathrm{n}} \rightarrow 0$ a.s. $/ / /$

To obtain convergence of weighted sums of random variables which are not identically distributed, dominance of the random variable by an integrable random variable or in some other sense is not an unusual condition (see Chow (1966), Stout (1968), or Rohatgi (1971)). However, it is a troublesome restriction. The strength provided by the results of this section is in the relaxation of conditions on the weights $\left\{a_{n k}\right\}$. An examination of the weights which are of Type $(P)$ is provided in the next section.

\section{CONDITIONS ON THE WEIGHTS.}

In this section the conditions on the weights will be examined. In particular, the Type (P) sequences will be shown to be different from the weights used in previous results. A strong law of large numbers can be obtained from these results, but the sequence $\left\{\sum_{k=1}^{n} a_{n k}: n \geq 1\right\}$ need not be bounded in general.

The first example will consist of weights $\left\{a_{n k}\right\}$ which satisfy Conditions 
(1) $-(4)$ and $\sum_{k=1}^{n} a_{n k}^{2} \rightarrow 0$ as $n \rightarrow \infty$ but which do not satisfy the conditions of Theorem 4 of Stout (1968) or Theorem 1 of Chow (1966). Define

$$
a_{n k}=[\ln (n+1)]^{-\frac{1}{2}}-(1+\epsilon), k, n=1,2, \ldots,
$$

where $\epsilon>0$. Then (1) - (4) hold and for all $n$

$$
\begin{aligned}
\sum_{k=1}^{n} \quad a_{n k}^{2} & =[\ln (n+1)]^{-1} \sum_{k=1}^{n} \frac{1}{k^{2+2 \epsilon}} \\
& \leq[\ln (n+1)]^{-1} \sum_{k=1}^{\infty} \frac{1}{k^{2+2 \epsilon}} \rightarrow 0 \text { as } n \rightarrow \infty .
\end{aligned}
$$

However, $A_{n}=\sum_{k=1}^{\infty} a_{n k}^{2}=\frac{1}{\ln (n+1)} \sum_{k=1}^{\infty} \frac{1}{k^{2+2 \epsilon}}=\frac{1}{\ln (n+1)} \lambda$, where $\lambda=\sum_{n=1}^{\infty} \frac{1}{k^{2+2 \epsilon}}>0$. Thus, $\sum_{k=1}^{\infty} \exp \left(-\lambda / A_{n}\right)=\sum_{n=1}^{\infty} \exp [-\ln (n+1)]$

$=\sum_{n=1}^{\infty} \frac{1}{n+1}=\infty$, which does not satisfy the hypothesis of Stout's (1968) Theorem 4 or Chow's (1966) Theorem 1.

For the next example, define

$$
a_{n k}= \begin{cases}n^{-1} & \text { if } k=1,2, \ldots, n \\ k^{-3 / 4} & \text { if } k=n+1, n+2, \ldots .\end{cases}
$$

This sequence satisfies Conditions (1)-(4), and hence a strong law of large numbers is available from the results of Section 2. However, since the second moments of the random variables in Theorem 2 can be uniformly bounded, Kolmogorov's criterion is easily satisfied for the welghts $a_{n k}=\bar{n}^{-1}, 1 \leq k \leq n$, and convergence of $s_{n}$ follows immediately. Thus, these results are most useful when considering nonuniform weighting where $\left\{\sum_{k=1}^{n} a_{n k}: n \geq 1\right\}$ may be unbounded. For example, define 


$$
a_{n k}= \begin{cases}\frac{k^{\frac{3}{4}}}{n} & \text { if } k=1,2, \ldots, n \\ \left(\sum_{j=n+1}^{\infty} \frac{1}{j^{3 / 2}}\right)^{\frac{1}{2}} & \text { if } k=n+1 \\ 0 & \text { if } k>n+1 .\end{cases}
$$

This sequence is also of Type (P).

Conditions (1) and (2) imply that

$$
\sum_{k=1}^{\infty} a_{k \mathrm{k}}^{2} \leq \Gamma .
$$

Thus, Kronecker type arguments would suffice for weights $\left\{a_{n k}\right\}$ where the decrease down the diagonal of the summability matrix offsets the possible decrease along the rows, for example, if $a_{n, k+1} a_{k k} \geq a_{n k} a_{k+1, k+1}$. However, letting

$$
a_{n k}= \begin{cases}n^{-\frac{1}{2} \ln (k+7)} & \text { if } k=1, \ldots, n-1 \\ n^{-1} & \text { if } k=n \\ \left(\sum_{j=n+1}^{\infty} j^{-2}\right)^{\frac{1}{2}} & \text { if } k=n+1 \\ 0 & \text { if } k>n+1\end{cases}
$$

defines weights which are of Type $(P)$ but which have the property that for each $k$ there exists an $n$ such that $a_{n, k+1} a_{k k}<a_{n k} a_{k+1, k+1}$. Moreover,

$$
a_{n k}= \begin{cases}n^{-k} & \text { if } k=1, \ldots, n-1 \\ n^{-1} & \text { if } k=n \\ \left.\sum_{j=n+1}^{\infty} j^{-2}\right)^{\frac{1}{2}} & \text { if } k=n+1 \\ 0 & \text { if } k>n+1\end{cases}
$$

defines extremely nonuniform weights which are of Type $(P)$ but such that for every $n>2, a_{n, k+1} a_{k k}<a_{n k} a_{k+1, k+1}$ for each $k=1, \ldots, n-2$. 
An exhaustive discussion of the weights which are of Type (P) will not be presented, but it is important to note that this more general condition on the weights is balanced by the assumption of total generalized Gaussian nonidentically distributed random variables.

4. EXTENSION TO RANDOM ELEMENTS IN A BANACH SPACE.

In this section an extension of Theorem 2 for random elements in a Banach space will be obtained. The study of random elements in abstract spaces was inspired by the consideration of stochastic processes as random elements in appropriate spaces of functions [see, for example Mann (1951) and Billingsley (1968)], and various properties of random variables have been extended to random elements. In particular, laws of large numbers for random elements in abstract spaces have been studied extensively [see Padgett and Taylor (1973), for example, and Alf (1975a) for a more recent result]. Also, Alf (1975b) has extended the results of Jamison, Orey, and Pruitt (1965) to weighted sums of random elements in a Banach space.

Let $X$ denote a (real) separable Banarh space with norm $\|\cdot\|$, and let $(\Omega, F, P)$ be a probability space. A random element $V$ in $X$ is a measurable function (with respect to the smallest sigma-field generated by the open subsets of $X$ ) from $\Omega$ into $X$. The random elements $\left\{v_{n}\right\}$ in $X$ are said to be identically distributed if their induced probabilities on $X$ are the same. Further, $\left\{\mathrm{v}_{\mathrm{n}}\right\}$ are said to be $\underline{\text { independent }}$ if for every finite collection $\left\{B_{1}, \ldots, B_{k}\right\}$ of Borel subsets of $X$

$$
\mathrm{P}\left[\mathrm{V}_{1} \in \mathrm{B}_{1}, \ldots, \mathrm{v}_{\mathrm{k}} \in \mathrm{B}_{\mathrm{k}}\right]=\prod_{i=1}^{\mathrm{k}} \mathrm{P}\left[\mathrm{v}_{i} \in \mathrm{B}_{i}\right] .
$$

An expected value for a random element $V$ in $X$ will be defined by the Pettis integral. That is, $V$ has expected value $E V \in X$ if $f(E V)=E[f(V)]$ for every 
continuous linear functional $f$ on $X$.

A Schauder basis for a Banach space $X$ is a sequence $\left\{b_{i}\right\} \subset X$ such that for each $x \in X$ there exists a unique sequence of scalars $\left\{t_{i}\right\}$ satisfying $x=\lim _{n \rightarrow \infty} \sum_{i=1}^{n} t_{i} b_{i}$. A sequence of linear operators $\left\{U_{n}\right\}$ can be defined on $X$ by

$$
U_{n}(x)=\sum_{i=1}^{n} f_{i}(x) b_{i}, n=1,2, \ldots,
$$

for $x \in X$ where $f_{i}(x)=t_{i}$ is the $i$ th coordinate functional for the basis. For a Banach space $X$, the coordinate functionals are continuous linear functionals on $X$. Further, a sequence of linear operators $\left\{Q_{n}\right\}$ may be defined on $X$ by $Q_{n}(x)=x-U_{n}(x), x \in X, n=1,2, \ldots$. It is well-known that for a Banach space there exists a basis constant $m$ : $>0$ such that $\left\|U_{n}\right\| \leq m$ for all $n$, and hence ||$Q_{n}|| \leq m+1$ for all $n$, Marti (1969) and Wilansky (1964).

Theorem 3 extends Theorem 2 to Banach spaces which have Schauder bases. A definition is needed first.

DEFINITION 2. Let $\mathrm{V}$ be a random element in a Banach space $X$ which has a Schauder basis with coordinate functionals $\left\{f_{i}\right\}$. Then $V$ is coordinatewise totally generalized Gaussian if $\mathrm{f}_{1}(\mathrm{~V})$ is a totally generalized Gaussian random variable for each $i=1,2, \ldots$.

THEOREM 3. Let $X$ be a Banach space with a Schauder basis $\left\{b_{i}\right\}$ and let $\left\{v_{k}\right\}$ be independent random elements in $X$ such that $E_{k}=0$. Let $\left\{a_{n k}\right\}$ be a Type (P) sequence. Suppose that for each $k=1,2, \ldots, V_{k}$ is a coordinatewise totally generalized Gaussian random element (with respect to the basis $\left.\left\{b_{1}\right\}\right)$, that for sufficiently large positive integers $p$ the random variables $\left\{|| Q_{p}\left(V_{k}\right)||-E|| Q_{p}\left(V_{k}\right)|| f_{k=1}^{\infty}\right.$ are generalized Gaussian with parameters $\alpha_{k} \leq 2^{\frac{1}{2}}$, and that as $p \rightarrow \infty$ there exist constants $C_{p}, C_{p} \rightarrow 0$, such that 


$$
\begin{aligned}
& \sup _{n} \sum_{k=1}^{n} \quad a_{n k} E\left\|Q_{p}\left(V_{k}\right)\right\| \leq C_{p} \text {. If } \sum_{k=1}^{n} a_{n k}^{2} \rightarrow 0 \text { as } n \rightarrow \infty \text {, then as } n \rightarrow \infty \\
& \left\|s_{n}\right\|=\left\|\sum_{k=1}^{n} a_{n k} v_{k}\right\| \rightarrow 0 \text { almost surely. }
\end{aligned}
$$

PROOF. For each $\mathrm{n}$ and $\mathrm{p}$, write

$$
s_{n}=\sum_{k=1}^{n} a_{n k} v_{k}=\sum_{k=1}^{n} a_{n k} U_{p}\left(v_{k}\right)+\sum_{k=1}^{n} a_{n k} Q_{p}\left(v_{k}\right) .
$$

Let $p$ be a fixed positive integer and consider

$$
\begin{aligned}
\left\|\mathrm{u}_{\mathrm{p}}\left(\sum_{\mathrm{k}=1}^{\mathrm{n}} \mathrm{a}_{\mathrm{nk}} \mathrm{v}_{\mathrm{k}}\right)\right\| & =|| \sum_{i=1}^{p} \mathrm{f}_{i}\left(\sum_{k=1}^{\mathrm{n}} \mathrm{a}_{\mathrm{nk}} \mathrm{v}_{\mathrm{k}}\right) \mathrm{b}_{i} \| . \\
& \leq \sum_{i=1}^{p}\left|\sum_{k=1}^{n} \mathrm{a}_{n k} \mathrm{f}_{i}\left(\mathrm{v}_{\mathrm{k}}\right)\right| \cdot|| \mathrm{b}_{i} \|,
\end{aligned}
$$

where $\left\{f_{i}\right\}$ are the coordinate functionals for the basis. Now since $\left\|f_{i}\right\| \neq 0, i=1, \ldots, p$, for each $i=1,2, \ldots, p,\left\{f_{i}\left(v_{k}\right)\right\}_{k=1}^{\infty}$ is a sequence of independent random variables with $E\left[f_{i}\left(V_{k}\right)\right]=f_{i}\left(E V_{k}\right)=0$ for all k. Thus, by Theorem 2, since $f_{i}\left(V_{k}\right)$ is totally generalized Gaussian for each $k$ and $i=$ $1,2, \ldots, p, \sum_{k=1}^{n} a_{n k} f_{i}\left(v_{k}\right) \rightarrow 0$ almost surely as $n \rightarrow \infty$ for each $i$. Thus, from (4.2) for each $p$ there exists an event $\Omega_{p}$ with $P\left(\Omega_{p}\right)=0$ such that $\omega \notin \Omega_{\mathrm{p}}$ implies that for $\epsilon>0$ there is an integer $N_{1}$ so that for $n \geq N_{1}$

$$
\left\|\mathrm{u}_{\mathrm{p}}\left(\sum_{\mathrm{k}=1}^{\mathrm{n}} \mathrm{a}_{\mathrm{nk}} \mathrm{v}_{\mathrm{k}}(\omega)\right)\right\|<\frac{\epsilon}{3} \text {. }
$$

Now, consider $\left\|Q_{p}\left(\sum_{k=1}^{n} a_{n k} v_{k}\right)\right\|$. For each $p,\left\{Q_{p}\left(v_{k}\right)\right\}_{k=1}^{\infty}$ is a sequence of independent random elements since $Q_{p}$ is a continuous linear operator. Thus, $\left\{\left(|| Q_{p}\left(V_{k}\right)||-E|| Q_{p}\left(V_{k}\right)||\right)\right\}_{k=1}^{\infty}$ are independent random variables with zero means for each $p$. Thus, by Theorem 2 , since $\left\|Q_{p}\left(V_{k}\right)\right\|-$ $E|| Q_{p}\left(V_{k}\right) \|$ is (totally) generalized Gaussian for each $k$ (and sufficiently large $p$ ), as $n \rightarrow \infty$

$$
\sum_{k=1}^{n} a_{n k}\left[|| Q_{p}\left(v_{k}\right)\left\|-E|| Q_{p}\left(v_{k}\right)\right\|\right] \rightarrow 0
$$


almost surely.

Now,

$$
\begin{aligned}
\left\|Q_{p}\left(\sum_{k=1}^{n} a_{n k} v_{k}\right)\right\| & =\left\|\sum_{k=1}^{n} a_{n k} Q_{p}\left(v_{k}\right)\right\| \\
& \leq \sum_{k=1}^{n} a_{n k}\left[\left\|Q_{p}\left(v_{k}\right)\right\|-E\left\|Q_{p}\left(V_{k}\right)\right\|\right] \\
& +\sum_{k=1}^{n} a_{n k} E|| Q_{p}\left(v_{k}\right) \| .
\end{aligned}
$$

But, by hypothesis $\sup _{n} \sum_{k=1}^{n} a_{n k} E|| Q_{p}\left(V_{k}\right)|| \leq C_{p}$. For $e>0$ and a large (fixed) $p_{O}$, there exists an integer $N_{2}$ such that for $n \geq N_{2}$, $\sum_{k=1}^{n} a_{n k} E|| Q_{p_{0}}\left(V_{k}\right)||<\frac{\epsilon}{3}$. Also, for sufficiently large $p_{1} \geq p_{0}$ and $\omega \neq \Omega_{0}^{\prime}$, where $P\left(\Omega_{0}^{\prime}\right)=0$, there exists an integer $N_{3}$ such that $n \geq N_{3}$ implies from (4.4) that

$$
\sum_{k=1}^{n} a_{n k}\left[\left\|Q_{p}\left(V_{k}(\omega)\right)\right\|-E|| Q_{p}\left(V_{k}\right)||\right]<\frac{\epsilon}{3} .
$$

Therefore, from (4.1), (4.3), (4.5) and (4.6) for $\omega \notin \Omega_{0} \cup \Omega_{0}^{\prime}$, where $\Omega_{0}=\underset{p}{\stackrel{\infty}{\underline{U}}} \Omega_{\mathrm{p}}$, for $\mathrm{p} \geq \mathrm{p}_{1}$, and for $\mathrm{n} \geq \max \left\{\mathrm{N}_{1}, \mathrm{~N}_{2}, \mathrm{~N}_{3}\right\}$,

$$
|| s_{n}(\omega)\|\leq\| U_{p}\left(\sum_{k=1}^{n} a_{n k} v_{k}(\omega)\right)\left\|+|| Q_{p}\left(\sum_{k=1}^{n} a_{n k} v_{k}(\omega)\right)\right\|<\epsilon . \quad \| / \mid
$$

Theorem 3 gives convergence results for random elements which need not be identically distributed and which do not have restrictive moment conditions (see the results of Padgett and Taylor (1976), for example). Also, the conditions on the weights $\left\{a_{n k}\right\}$ are very general as indicated in Section 3. Moreover, the uniform dominance in probability of Rohatgi (1971) and Padgett and Taylor (1974) is eliminated by the modified generalized Gaussian type of condition. Finally, if $X=R^{n}$ with the usual norm, then Theorem 3 gives a convergence theorem for $n$-dimensional random vectors, and since $f_{i}(x)=0$ 
for $1>n, x \in R^{n}, Q_{p}(x)=0$ for $p>n$, and only Inequality (4.3) is needed. These results may be applied to stochastic processes. For example,

Theorem 3 may be applied to sequences of separable Wiener processes on $[0,1]$ (or $[0, \mathrm{~T}]$ ) since such processes may be considered as random elements in the Banach space C $[0,1]$ [Billingsley (1968)] which has a Schauder basis.

\section{REFERENCES}

1. Alf, Carol. Rates of convergence for the laws of large numbers for independent Banach-valued random variables, J. Multivariate Anal. 5 (1975a) 322-329.

2. Alf, Carol. Convergence of weighted sums of independent Banach-valued random variables (abstract), I. M. S. Bulletin 4 (1975b) 139.

3. Billingsley, P. Convergence of Probability Measures, Wiley, New York, 1968 .

4. Chow, Y. S. Some convergence theorems for independent random variables, Ann. Math. Statist. 37 (1966) 1482-1493.

5. Chow, Y. S. and Lai, T. L. Limiting behavior of weighted sums of independent random variables, Ann. Prob. 1 (1973) 810-824.

6. Hsu, P. L. and Robbins, H. Complete convergence and the law of large numbers, Proc. Nat. Acad. Sci. U. S. A. 33 (1947) 25-31.

7. Jamison, B., Orey, S. and Pruitt, W. Convergence of weighted averages of independent random variables, $Z$. Wahr. Verw. Gebiete 4 (1965) 4044 .

8. Lai, T. L. Control charts based on weighted sums, Ann. Statist. $\underline{2}$ (1974) 134 .

9. Mann, H. B. On the realization of stochastic processes by probability distributions in function spaces, Sankhya 11 (1951) 3-8.

10. Mart1, J. T. Introduction to the Theory of Bases, Springer-Verlag, New York, 1969.

11. Padgett, W. J. and Taylor, R. L. Laws of Large Numbers for Normed Linear Spaces and Certain Fréchet Spaces, Lecture Notes in Mathematics, Vol. 360, Springer-Verlag, Berlin-Heidelberg-New York, 1973. 
12. Padgett, W. J. and Taylor, R. L. Convergence of weighted sums of random elements in Banach spaces and Fréchet spaces, Bull. Inst. Math., Academia Sinica 2 (1974) 389-400.

13. Padgett, W. J. and Taylor, R. L. Almost sure convergence of weighted sums of random elements in Banach spaces, Probability in Banach Spaces, Oberwolfach, 1975, A. Beck, Ed. Lecture Notes in Mathematics, Vo1. 526, Springer-Verlag, Berlin, 187-202, 1976.

14. Rohatgi, V. K. Convergence of weighted sums of independent random variables, Proc. Cambridge Philos. Soc. 69 (1971) 305-307.

15. Stout, William F. Some results on the complete and almost sure convergence of linear combinations of independent random variables and martingale differences, Ann. Math. Statist. 39 (1968) 1549-1562.

16. Tucker, H. G. A Graduate Course in Probability, Academic Press, New York, 1967.

17. Wilansky, A. Functional Analysis, Blaisde11, New York, 1964 . 


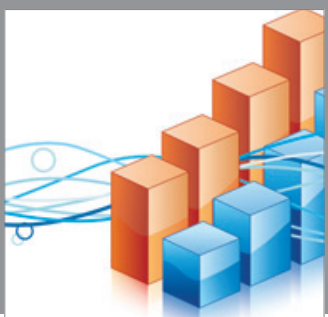

Advances in

Operations Research

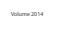

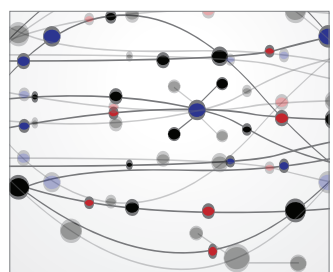

\section{The Scientific} World Journal
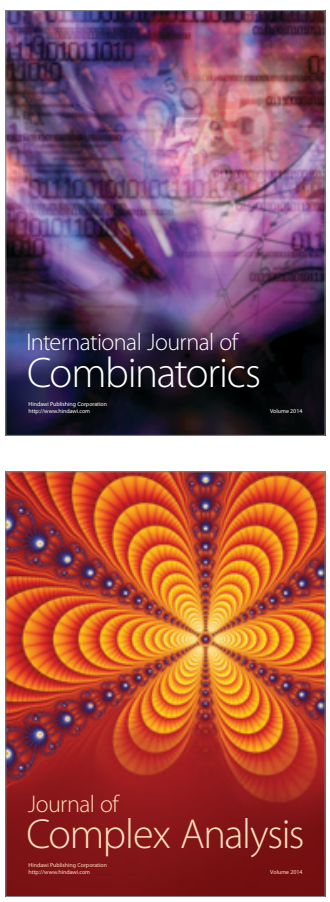

International Journal of

Mathematics and

Mathematical

Sciences
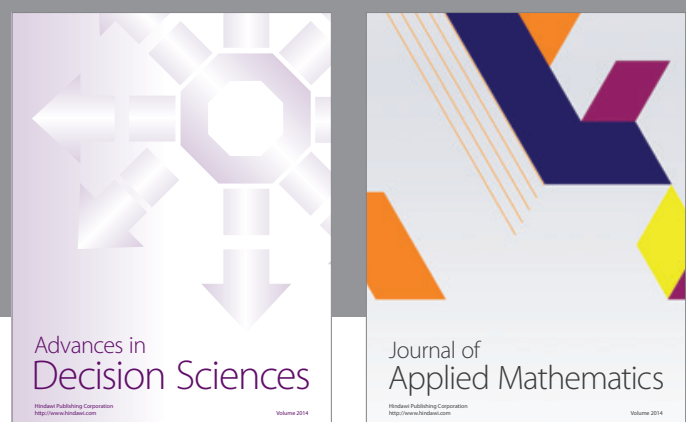

Journal of

Applied Mathematics
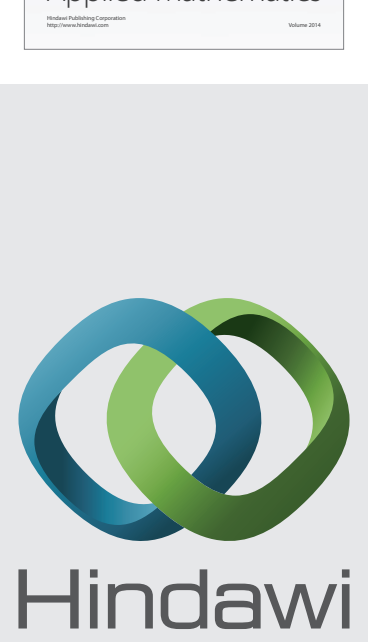

Submit your manuscripts at http://www.hindawi.com
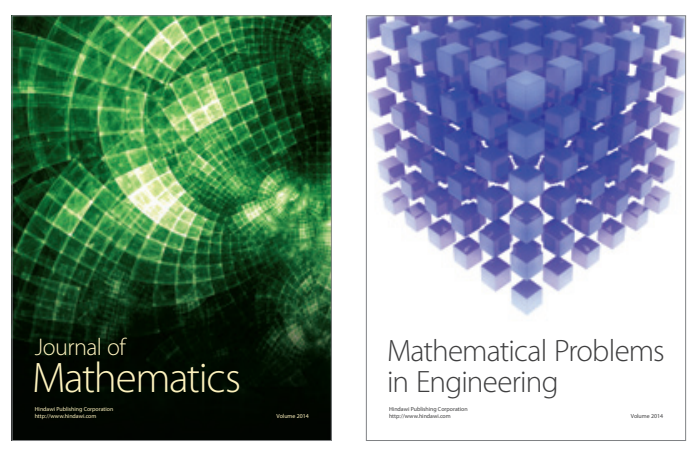

Mathematical Problems in Engineering
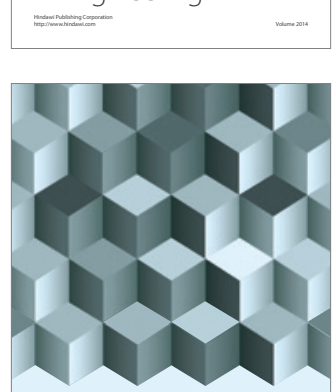

Journal of

Function Spaces
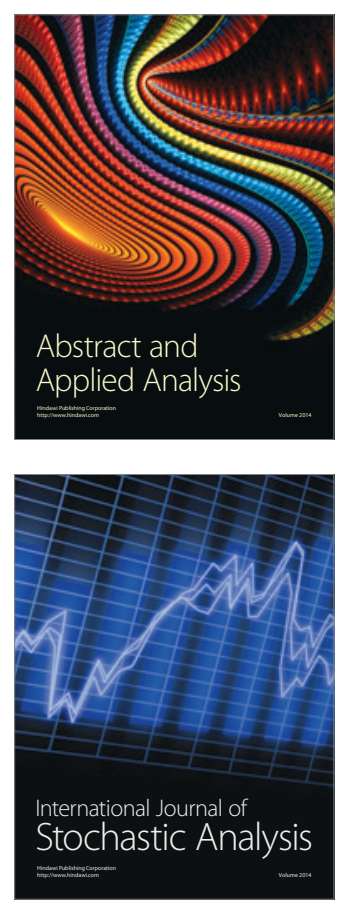

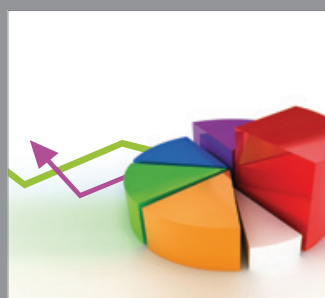

ournal of

Probability and Statistics

Promensencen
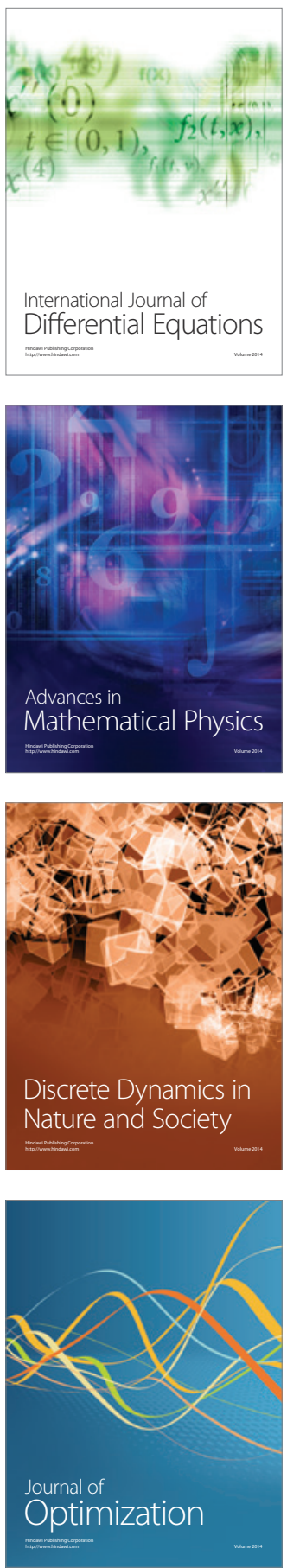